\title{
Application of MADM methods as MOORA and WEDBA for ranking of FMS flexibility
}

\author{
Vineet Jain $^{a^{*}}$ and Puneeta Ajmera ${ }^{\text {b }}$
}

\begin{tabular}{l}
${ }^{a}$ Department of Mechanical Engineerin \\
${ }^{b}$ Department of Hospital Administration \\
\hline C H R O N I C L E \\
\hline Article history: \\
Received: October 19, 2018 \\
Received in revised format: Octo- \\
ber 25, 2018 \\
Accepted: December 26, 2018 \\
Available online: \\
December 26, 2018 \\
\hline Keywords: \\
FMS \\
Flexibility \\
Ranking \\
MADM \\
MOORA \\
AHP WEDBA \\
Entropy
\end{tabular}

\section{Introduction}

FMS is a flexible manufacturing system. It is 'flexible' as it has the ability to produce a range of unalike part styles simultaneously at the different workstations, in reaction of sudden market demands (Jain \& Raj, 2015b). Jain and Raj (2017) discussed that "flexible manufacturing system (FMS) is an integrated, computer-controlled complex arrangement of automated material handling devices and numerically controlled (NC) machine tools that can simultaneously process medium sized volumes of a variety of part types". According to Chen and Chung (1996) "flexibility refers to the ability of the manufacturing system to respond quickly to changes in part demand and part mix" and according to Das (1996) "ability of a system or facility to adjust to changes in its internal or external environment". In previous research, multiple-attribute-decision-making (MADM) methods were discussed for sorting of flexibility by AHP, TOPSIS, Improved PROMETHEE, Modified TOPSIS, VIKOR, SAW, WPM and GTMA (Jain \& Raj,

\footnotetext{
* Corresponding author. Tel.: +918901510570

E-mail address: vjdj2004@gmail.com (V. Jain)

(C) 2019 by the authors; licensee Growing Science, Canada. doi: $10.5267 /$ j.ijdns.2018.12.003

Flexibility has been cited as a key factor to enhance the performance of flexible manufacturing system (FMS). The main aim of this paper is to rank the flexibility of FMS. The ranking decisions are complex in the manufacturing field to analyze a number of alternatives based on a set of some attibutes. In this research, two MADM methods i.e. MOORA (i.e. multi-objective optimization on the basis of ratio analysis) and weighted Euclidean distance based approach (WEDBA) are used for ranking of flexibility in FMS for new part development. MOORA approach can give in WEDBA, integrated attribute weights are used for evaluation which included the subjective and objective weights of attributes. Objective weights are calculated by entropy method and subjective weights are calculated by analytic hierarchy process. MOORA is applied in two ways i.e. fit based and reference point analysis. Ranking of fifteen flexibility of FMS done on the basis shows that product flexibility has the top most flexibility in fifteen flexibilities and programme flexibility has the least impact in fifteen flexibilities. 
2013b, 2013c, 2014a, 2015a, 2015b). Jain and Raj (2014a) used VIKOR method for evaluation of flexibility based on subjective weights as calculated by AHP method. Jain and Raj (2015b) used modified TOPSIS method based on subjective weights. Generally, subjective weights were used for MADM methods. But objective weights may be considered for taking any decision. So, an integrated weights are used in this paper which came from subjective and objective weights. Objectives weights are calculated from entropy method and subjective weights taken by AHP method.

In this research, ranking of flexibility of FMS is done by MADM methods as MOORA and WEDBA based on fifteen variables which effect the flexibility of FMS. These methods are easy to use. Chakraborty (2011) also stated that "in MOORA less computational time required for performing mathematical calculations". According to some researchers, (Jain \& Raj, 2013a, 2013b, 2013c, 2014a, 2014b, 2015a, 2015b, 2015c, 2016; Jain \& Raj, 2018; Raj et al., 2012), fifteen flexibilities and fifteen variables which effect flexibility of FMS are shown in Fig. 1 and Fig. 2 respectively.

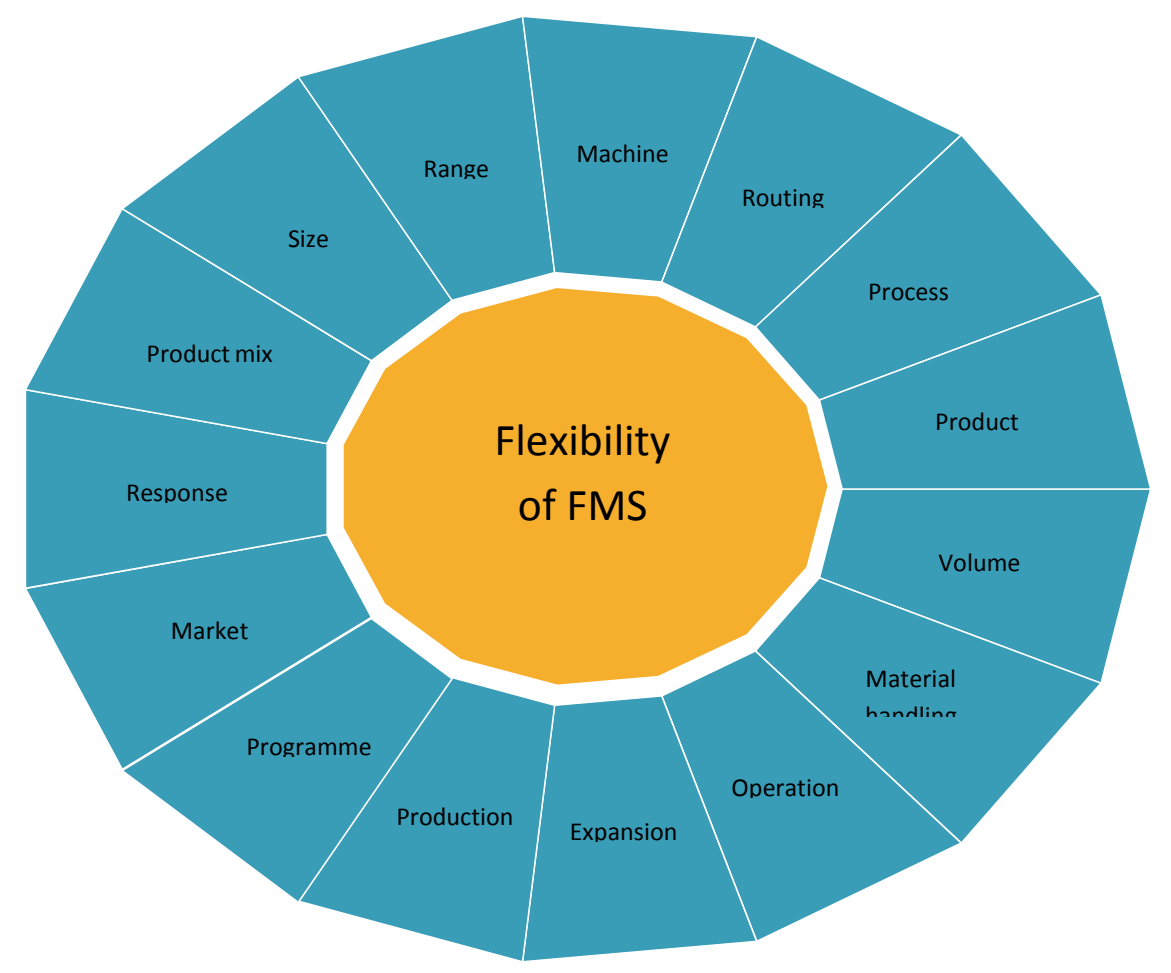

Fig. 1. Flexibility of FMS

The main objectives of this research are as follows:

To find the objective and integrated weights of the attributes

$>$ Ranking of flexibility based on variables by using weighted Euclidean distance based approach and MOORA methodologies.

In this paper, an overview of weighted Euclidean distance based approach methodology discussed in section 2. In section 3, an overview of MOORA MADM Method is presented. In section 4, analysis of ranking of flexibilities by weighted Euclidean distance based approach is discussed. Analysis of ranking of flexibility of FMS by MOORA is discussed in section 5. Discussion and conclusion are discussed in section 6 . 


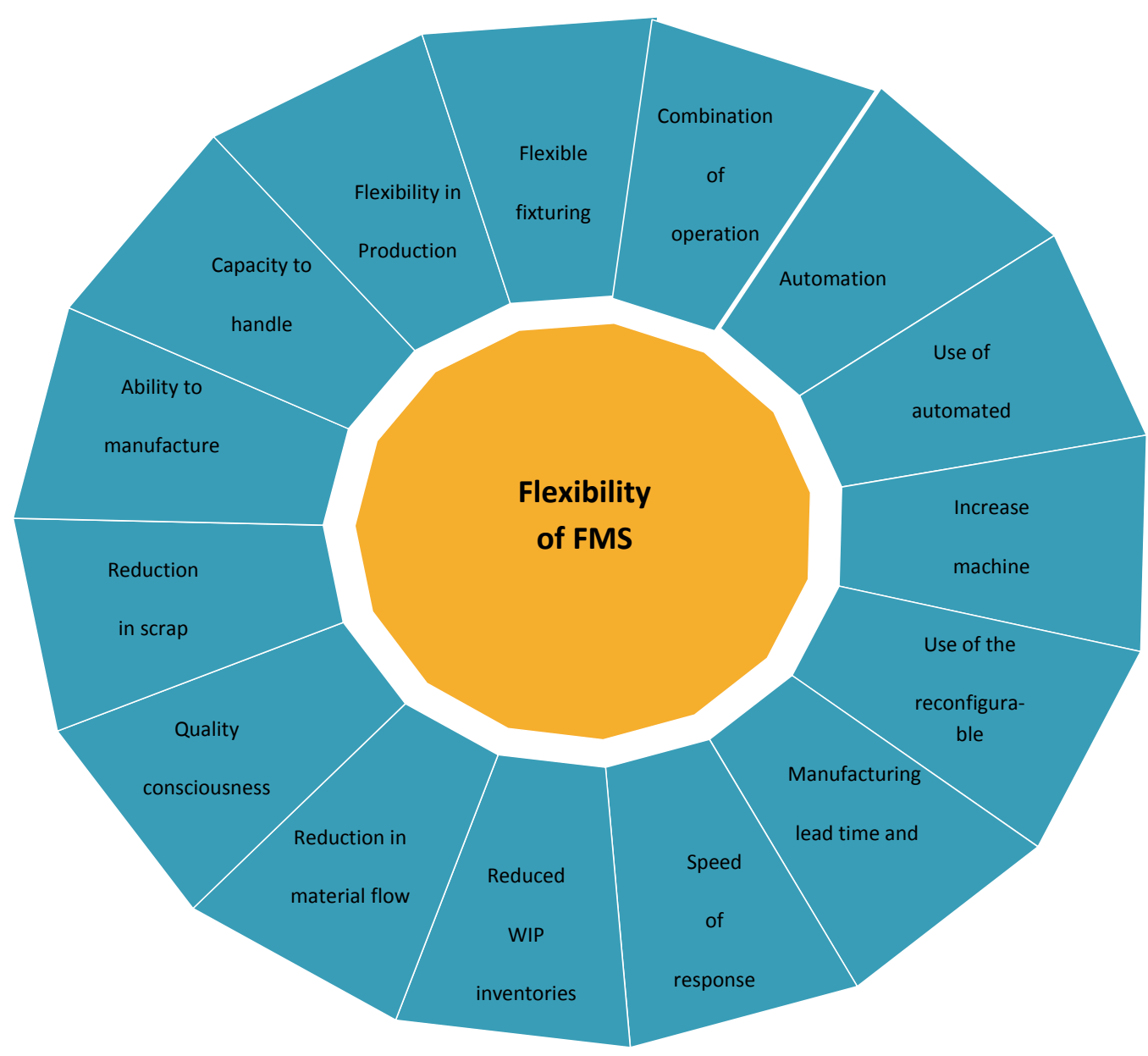

Fig. 2. Variables which effect the flexibility of FMS

\section{Weighted Euclidean distance based approach (WEDBA) methodology}

Dattorro (2005) and Gower (1982) established the Euclidean distance concept in the field of Mathematics. This approach is based on the weighted distance of alternatives from the most and least favorable situations respectively. The ideal point is represented as the most favorable situation and the antiideal point is represented as the least favorable situation (Rao, 2013; Venkata Rao \& Singh, 2012). Three types of attribute weights are considered in WEDBA method i.e. 1- objective weights, 2- subjective weights and 3- integrated weights. An overview of the WEDBA method is described below (Venkata Rao \& Singh, 2011) :

Step 1: To find the Decision matrix

The collection of attribute data for each alternative is a decision matrix. In this decision matrix, $\mathrm{M}$ is the alternatives and $\mathrm{N}$ is the attributes, which is expressed as the $\mathrm{i}^{\text {th }}$ alternative i.e. $\mathrm{i}(\mathrm{i}=1,2,3, \ldots, \mathrm{M})$ and for alternative $\mathrm{j}(\mathrm{j}=1,2,3, \ldots, \mathrm{N})$. The standard form of decision matrix is given by Eq. (1). 


$$
\mathrm{D}_{M X N}=\begin{array}{r}
D_{1} \\
D_{2} \\
D_{3} \\
- \\
- \\
D_{M}
\end{array}\left[\begin{array}{cccccc}
\mathrm{D}_{1} & D_{2} & \mathrm{D}_{3} & - & - & \mathrm{D}_{N} \\
\mathrm{~d}_{11} & d_{12} & \mathrm{~d}_{13} & - & - & d_{1 \mathrm{~N}} \\
d_{21} & \mathrm{~d}_{22} & d_{23} & - & - & d_{2 \mathrm{~N}} \\
d_{31} & d_{32} & \mathrm{~d}_{33} & - & - & d_{3 N} \\
- & - & - & - & - & - \\
- & - & - & - & - & - \\
d_{M 1} & d_{M 2} & \mathrm{~d}_{M 3} & - & - & \mathrm{d}_{\mathrm{MN}}
\end{array}\right]
$$

Chen and Hwang (1992) proposed an approach to solve more than 10 alternatives and they proposed first converts linguistic terms into fuzzy numbers and then the fuzzy numbers into crisp scores. The attributes are represented in linguistic terms and converted into corresponding crisp scores according to 11 point scale as given in Fig. 3 and Table 1 below (Jain \& Raj, 2013c).

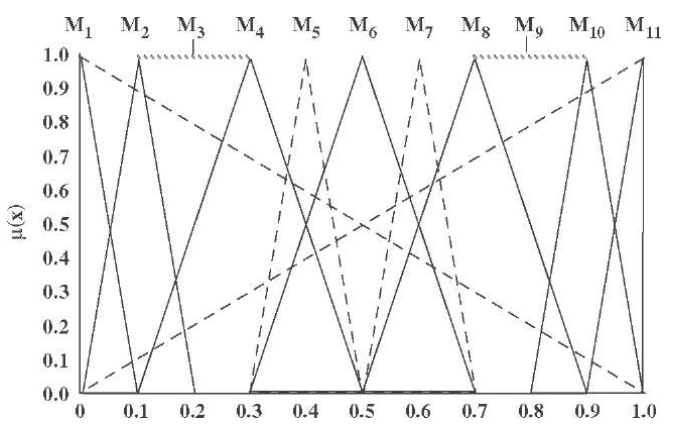

Fig. 3. Linguistic terms into their corresponding fuzzy (Jain \& Raj, 2013)

Table 1

Conversion of linguistic terms into fuzzy scores (11-point scale) (Jain \& Raj, 2013c)

\begin{tabular}{ccc}
\hline Linguistic term & Fuzzy no. & Crisp no. \\
\hline Exceptionally low & $\mathrm{M}_{1}$ & 0.045 \\
Extremely low & $\mathrm{M}_{2}$ & 0.135 \\
Very low & $\mathrm{M}_{3}$ & 0.255 \\
Low & $\mathrm{M}_{4}$ & 0.335 \\
Below average & $\mathrm{M}_{5}$ & 0.410 \\
Average & $\mathrm{M}_{6}$ & 0.500 \\
Above average & $\mathrm{M}_{7}$ & 0.590 \\
High & $\mathrm{M}_{8}$ & 0.665 \\
Very high & $\mathrm{M}_{9}$ & 0.745 \\
Extremely high & $\mathrm{M}_{10}$ & 0.865 \\
Exceptionally high & $\mathrm{M}_{11}$ & 0.955 \\
\hline
\end{tabular}

Step 2: To standardization of attribute data

In this step, standardization of attribute data is take place. The obtained values from the standardized process called as standard scores. It has value 0 and 1 i.e. standard score is mean of zero and a variance of 1 (i.e., standard deviation equals to 1). The standardized decision matrix $D_{M \times N}^{*}$ is given as follows: 


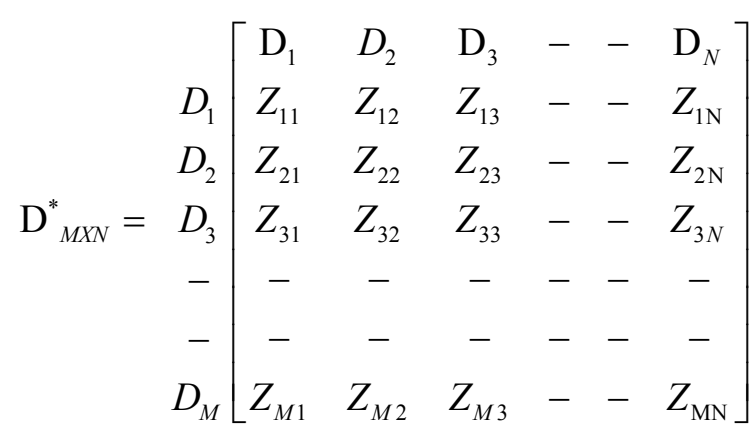

where

$$
\begin{aligned}
& Z_{i j}=\frac{x_{i j}-\mu_{j}}{\sigma_{j}} \\
& \left.x_{i j}=\frac{d_{i j}}{\max _{j}\left(d_{i j}\right)} ; \quad \text { if } \mathrm{j} \text { th attribute is beneficial }\right) \\
& x_{i j}=\frac{\min _{j}\left(d_{i j}\right)}{d_{i j}} ; \quad(\text { if } \mathrm{j} \text { th } \text { attribute is non beneficial }) \\
& \mu_{j}=\frac{1}{M} \sum_{i=1}^{M} x_{i j} \\
& \sigma_{j}=\sqrt{\frac{\sum_{i=1}^{M}\left(x_{i j}-\mu_{j}\right)^{2}}{M}}
\end{aligned}
$$

$Z_{i j}$ is the standardized value of $x_{i j}, \mu_{j}$ is the expected value or mean value for $\mathrm{j}^{\text {th }}$ attribute and $\sigma_{j}$ is the standard deviation for the attribute $\mathrm{j}$.

Step 3: To find the Ideal and anti-ideal points

The attribute values which is most desired is the ideal point and which is not desired i.e. least desirable is the anti- ideal point. These point are shown by $\mathrm{a}^{*}$ and $\mathrm{b}^{*}$ for ideal and anti-ideal point respectively.

$$
a^{*}=\left\{a_{j}^{*}\right\} \text { and } b^{*}=\left\{b_{j}^{*}\right\} \text { where } j=\{1,2, \ldots . . N\}
$$

Step 4: To find the attribute weights

The attributes weights may be used by the researcher, depends upon the application condition. It may be subjective or objective or combination of subjective and objective i.e. integrated weights.

a) To find the objective weights of the attributes

To calculate objective weights, entropy method is suggested in this research work. Entropy is a measure of uncertainty in the information formulated using probability theory. It is based in information theory which assigns a small weight to an attribute if it has similar attribute values across alternatives, because such attribute does not help in differentiating alternatives.

The following are the steps to determine objective weight of attributes by entropy method as given below:

The Entopy value $\left(E_{j}\right)$ 
The amount of decision information contained in the decision matrix and associated with each attribute can be measured by the entropy value $\left(\mathrm{E}_{\mathrm{j}}\right)$ as:

$$
E_{j}=\frac{-\left(\sum_{i=1}^{M} p_{i j} \ln p_{i j}\right)}{\ln M} ;(j=1,2, \ldots M)
$$

Where

$$
p_{i j}=\frac{d_{i j}}{\sum_{k=1}^{M} d_{k j}}
$$

$\mathrm{d}_{\mathrm{ij}}$ is the value of attribute $\mathrm{j}$ for alternative $\mathrm{i}$ and $\mathrm{N}$ is the number of alternatives.

\section{The degree of divergence $\left(d d_{j}\right)$}

The degree of divergence $\left(\mathrm{dd}_{\mathrm{j}}\right)$ of the average intrinsic information contained by each attribute can be calculated as,

$$
d d_{j}=1-E_{j},(1 \leq j \leq N)
$$

The more divergent the performance ratings $p_{i j}(i=1,2, \ldots, M)$ for the attribute $j$, the higher its corresponding $\mathrm{dd}_{\mathrm{j}}$, and the more important the attribute $\mathrm{j}$ for the decision making problem under consideration (R.V. Rao, 2007).

\section{The objective weights}

The objective weight for each attribute $\mathrm{j}$ is thus given by

$$
w_{i j}=\frac{d d_{j}}{\sum_{j=1}^{N} d d_{j}}
$$

b) To find the subjective weights of the attributes

The subjective weights may be calculated by analytic hierarchy process (AHP). So, these weights are calculated by AHP method (Saaty, 2000). A set of weights, $w_{j}$ (for $j=1,2, \ldots . ., N$ ) such that $\sum w_{j}=1$ may be decided upon. Jain and Raj (2013c) used AHP method to calculate these weights. So, these weights are taken here.

c) To find the integrated weights of the attributes

Integrated weights are the combination of subjective and objective weights. It is used when the researcher want to use both the weights i.e. objective and subjective weights of the attributes. The integrated weights are obtained using the formula given below. 


$$
w_{j}^{I}=\frac{w_{j}^{o} \times w_{j}^{s}}{\sum_{k=1}^{N} w_{k}^{o} \times w_{k}^{s}}
$$

where $w_{j}^{o}$ is the objective weight, $w_{j}^{s}$ is the subjective weight and $w_{j}^{I}$ is the integrated weight of the $j^{\text {th }}$ attribute.

Step 5: To find the weighted Euclidean distance (WED), index score and ranking

The WEDBA method is based on the Euclidean distance i.e. the shortest distance between two points. The top ranked alternative is closest to the ideal solution and least ranked is the farthest from the antiideal solution. The overall performance index score of an alternative is determined by its Euclidean distance to ideal solution and anti- ideal solutions (Venkata Rao \& Singh, 2011, 2012). WED between an alternative $i$ and ideal point $\mathrm{a}^{*}$ is denoted by $\mathrm{WED}_{\mathrm{i}}^{+}$and between an alternative $i$ and anti-ideal point $\mathrm{b}^{*}$ is denoted by WED ${ }^{-}$.

$$
\begin{aligned}
& W E D_{i}^{+}=\left[\sum_{j=1}^{N}\left\{w_{j}\left(Z_{i j}-a_{j}^{*}\right)\right\}^{2}\right]^{1 / 2} \text { for }(i=1,2, \ldots M) \\
& W E D_{i}^{-}=\left[\sum_{j=1}^{N}\left\{w_{j}\left(Z_{i j}-b_{j}^{*}\right)\right\}^{2}\right]^{1 / 2} \text { for }(i=1,2, \ldots M)
\end{aligned}
$$

The index score is calculated using following Eq. (16).

$$
\text { Index } \text { Score }_{i}=\frac{W E D_{i}^{-}}{W E D_{i}^{+}+W E D_{i}^{-}}
$$

The index score represents the relative closeness of a particular alternative to the ideal solution. The higher the index score for a particular alternative, the closer the alternative to the ideal solution. The alternative for which the value of index score is highest is the best choice for the considered decision making problem.

\section{MOORA Methodology}

In this section, MOORA MADM approach is presented for the ranking of flexibility of FMS. The MOORA approach refers to the ratio analysis and reference point approach. This approach is mainly used for multi-attribute optimization. This approach was presented by W. K. Brauers (2004). The researcher used MOORA in different ways and it is shown in Table 2 (Attri \& Grover, 2014; Brauers, 2013; Brauers \& Zavadskas, 2009, 2010, 2011; Chakraborty, 2011; Chand et al., 2014; Gadakh, Shinde, \& Khemnar, 2013; Ginevičius et al., 2010; Karande \& Chakraborty, 2012).

Table 2

MOORA applications found in literature

\begin{tabular}{ccc}
\hline Sr. No. & Name of the authors & Application \\
\hline 1 & Jain (2018) & FMS performance variables \\
2 & Attri and Grover (2014) & Decision making in production system life cycle \\
3 & Chand et al. (2014) & Risks assessment in supply chain \\
4 & Brauers (2013) & Decision making of seaport \\
5 & Gadakh et al. (2013) & Welding process parameters optimization \\
6 & Karande and Chakraborty (2012) & Selection of material \\
7 & Chakraborty (2011) & Decision making in manufacturing environment \\
8 & Brauers and Zavadskas (2011) & Decision to buy property by a bank loan \\
9 & Brauers and Zavadskas (2010) & Project management economics \\
\hline
\end{tabular}




\begin{tabular}{ccc}
\hline 10 & Ginevičius et al. (2010) & Regional development in a country \\
11 & Brauers and Zavadskas (2009) & To test the facilities sector \\
\hline
\end{tabular}

MOORA MADM method is applied according to the Karande and Chakraborty (2012), Attri and Grover (2014) and Jain (2018) as given below. Diagram of MOORA is shown in Fig. 4.

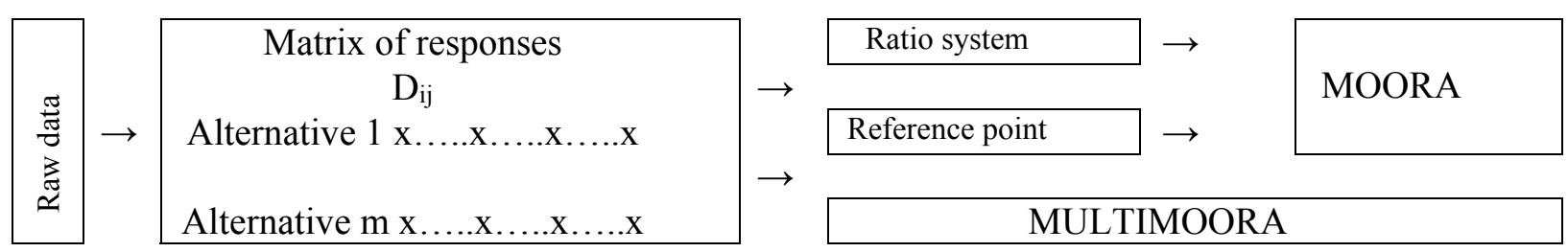

Fig. 4. Diagram of MOORA (Brauers 2013)

\section{(a) Ratio System Approach}

The following steps are involved in the ratio system approach as given below:

Step 1: Formulate the decision matrix:

MOORA approach starts with the creation of decision matrix comprising of value of various alternatives relating to different attributes as per equation 1 .

The attributes are represented in linguistic terms and converted into corresponding crisp scores according to 11 point scale as shown in Fig. 3 and Table 1.

Step 2: Normalize the decision matrix:

In this step of MOORA approach, the decision matrix is normalized for making the decision matrix dimensionless. Normalization of the decision matrix is carried out by using the following equation (Karande \& Chakraborty, 2012):

$$
N_{i j}=\frac{D_{i j}}{\sum_{i=1}^{m} D_{i j}}
$$

Here, $\mathrm{N}_{i j}=$ Normalized value i.e. dimensionless number (in 0, 1 interval), which describes the normalized performance value.

\section{Step 3: Classification of attributes:}

In this step, attributes are classified into two categories viz. beneficial attribute and non-beneficial attribute. Beneficial attributes are those attributes whose greater values are essential; while non-beneficial attributes are those attributes whose lower values are essential.

Step 4: Computation of assessment value:

The assessment value of each alternative is calculated by the following equation:

$$
y_{i}=\sum_{j=1}^{g} N_{i j}-\sum_{j=g+1}^{n} N_{i j}
$$

Here, $y_{i}$ is the assessment value of $i^{t h}$ alternative with regard to all the attributes, $g$ is the number of 
attribute to be maximized, $(n-g)$ is the number of attribute to be minimized.

In Eq. (18), all the normalized performances are totalled for the beneficial attributes and deducted for the non-beneficial attributes. The assessment value can be positive or negative.

In some specific cases, attributes may be more significant than one another. To take this effect into consideration, weight of attributes is considered. The weights of attributes are generally computed by using the AHP approach. When the weights of attributes are taken into consideration, then equation 3 becomes:

$$
y_{i}^{*}=\sum_{j=1}^{g} w_{i} N_{i j}-\sum_{j=g+1}^{n} w_{i} N_{i j}
$$

where $y^{*}{ }_{i}$ is the assessment value of $i^{\text {th }}$ alternative with regard to all the attributes which include weights of attributes, $w_{i}$ is the weight of $j^{\text {th }}$ attribute.

Step 5: Determine the relative importance of the attributes i.e. weights of attributes.

According to Saaty (2000) "construct a pair-wise comparison matrix using a scale of relative importance".

The attribute weights $\left(\mathrm{w}_{\mathrm{j}}\right)$ by Eq. (20) and Eq. (21).

$$
G M_{J}=\left[\prod_{i=1}^{N} d_{i j}\right]^{\frac{1}{N}}
$$

and

$$
W_{J}=G M_{j} / \sum_{j=0}^{N} G M_{j}
$$

Step 5: Selection of best alternative:

The alternative having the maximum assessment value is considered as the best alternative.

\section{(b) Reference Point Approach}

The steps involved in the reference point approach are given below:

Step 1: Formulate the decision matrix:

In this step, decision matrix is constructed as in the ratio system approach.

Step 2: Normalize the decision matrix:

Here, the decision matrix is normalized to make it dimensionless. It is normalized in the similar manner as in ratio system approach.

Step 3: Classification of attributes:

In this step, attributes are categorized into two categories viz. beneficial attributes and non-beneficial attributes.

Step 4: Computation of reference point:

In this step, a reference point is deduced from the normalized matrix. The computation of reference point depends upon the beneficial and non-beneficial attributes.

The reference point in case of beneficial attribute is the maximum value while in case of non-beneficial attribute, it is minimum value.

Step 5: Computation of deviation from reference point: 
The deviation of an attribute value from its set reference point $\left(r_{i}\right)$ is calculated by following equation:

$$
r_{i}-N_{i j}
$$

Step 6: Computation of performance index:

The best alternative in the reference point approach will have maximum values in case of beneficial attributes along with the minimum values in case of non-beneficial attributes.

In practical situations, it is not likely all the times that a specific alternative will have all of the maximum values in the case of beneficial attributes and minimum values in the case of non-beneficial attributes. In such situations, there will be deviance from the reference point series. The deviation is computed by using the following equation (Brauers \& Zavadskas, 2006):

$$
P_{i}=\min _{i}\left\{\max _{j}\left|r_{i}-N_{i j}\right|\right\}
$$

where $P_{i}=$ performance index

Step 7: Selection of best alternative:

The best alternative will have the total minimum deviance from the reference point series i.e. minimum value of $P_{i}$.

\section{Ranking of flexibility by WEDBA}

In this section, the ranking of flexibility is carried out by WEDBA is given below.

Step 1 Decision matrix

The measures of attributes for the flexibility ranking are given in Table 3 . The values of are given in fuzzy linguistic terms. The conversion of linguistic data into corresponding crisp values is done using the eleven point fuzzy scale and the corresponding equivalent crisp values of attribute are shown in Table 4.

Table 3

\begin{tabular}{|c|c|c|c|c|c|c|c|c|c|c|c|c|c|c|c|}
\hline & 1 & 2 & 3 & 4 & 5 & 6 & 7 & 8 & 9 & 10 & 11 & 12 & 13 & 14 & 15 \\
\hline 1 & $\begin{array}{l}\text { Ex- } \\
\text { tremely } \\
\text { high }\end{array}$ & High & High & $\begin{array}{l}\text { Aver- } \\
\text { age }\end{array}$ & $\begin{array}{c}\text { Above } \\
\text { average }\end{array}$ & $\begin{array}{l}\text { Aver- } \\
\text { age }\end{array}$ & $\begin{array}{c}\text { Below } \\
\text { average }\end{array}$ & $\begin{array}{c}\text { Above } \\
\text { average }\end{array}$ & High & High & $\begin{array}{c}\text { Above } \\
\text { average }\end{array}$ & Low & $\begin{array}{l}\text { Very } \\
\text { low }\end{array}$ & Average & $\begin{array}{c}\text { Below } \\
\text { average }\end{array}$ \\
\hline 2 & $\begin{array}{c}\text { Below } \\
\text { average }\end{array}$ & $\begin{array}{c}\text { Below } \\
\text { average }\end{array}$ & High & $\begin{array}{l}\text { Aver- } \\
\text { age }\end{array}$ & $\begin{array}{l}\text { Very } \\
\text { low }\end{array}$ & $\begin{array}{l}\text { Aver- } \\
\text { age }\end{array}$ & $\begin{array}{c}\text { Above } \\
\text { average }\end{array}$ & $\begin{array}{c}\text { Above } \\
\text { average }\end{array}$ & $\begin{array}{c}\text { Below } \\
\text { average }\end{array}$ & $\begin{array}{c}\text { Below } \\
\text { average }\end{array}$ & High & $\begin{array}{c}\text { Above } \\
\text { average }\end{array}$ & Average & $\begin{array}{c}\text { Below } \\
\text { average }\end{array}$ & Average \\
\hline 3 & High & Average & $\begin{array}{c}\text { Above } \\
\text { average }\end{array}$ & $\begin{array}{c}\text { Above } \\
\text { average }\end{array}$ & Average & $\begin{array}{l}\text { Aver- } \\
\text { age }\end{array}$ & $\begin{array}{c}\text { Below } \\
\text { average }\end{array}$ & $\begin{array}{l}\text { Above } \\
\text { average }\end{array}$ & Average & $\begin{array}{l}\text { Aver- } \\
\text { age }\end{array}$ & $\begin{array}{c}\text { Above } \\
\text { average }\end{array}$ & $\begin{array}{c}\text { Aver- } \\
\text { age }\end{array}$ & Average & $\begin{array}{c}\text { Below } \\
\text { average }\end{array}$ & Average \\
\hline 4 & $\begin{array}{l}\text { Very } \\
\text { high }\end{array}$ & $\begin{array}{c}\text { Ex- } \\
\text { tremely } \\
\text { high }\end{array}$ & High & $\begin{array}{c}\text { Above } \\
\text { average }\end{array}$ & $\begin{array}{c}\text { Below } \\
\text { average }\end{array}$ & $\begin{array}{l}\text { Aver- } \\
\text { age }\end{array}$ & $\begin{array}{l}\text { Below } \\
\text { average }\end{array}$ & $\begin{array}{c}\text { Above } \\
\text { average }\end{array}$ & $\begin{array}{c}\text { Above } \\
\text { average }\end{array}$ & $\begin{array}{l}\text { Aver- } \\
\text { age }\end{array}$ & High & $\begin{array}{c}\text { Below } \\
\text { average }\end{array}$ & $\begin{array}{c}\text { Below } \\
\text { average }\end{array}$ & Average & $\begin{array}{c}\text { Below } \\
\text { average }\end{array}$ \\
\hline 5 & $\begin{array}{c}\text { Below } \\
\text { average }\end{array}$ & $\begin{array}{c}\text { Below } \\
\text { average }\end{array}$ & $\begin{array}{c}\text { Below } \\
\text { average }\end{array}$ & $\begin{array}{l}\text { Aver- } \\
\text { age }\end{array}$ & Average & $\begin{array}{c}\text { Above } \\
\text { average }\end{array}$ & $\begin{array}{c}\text { Above } \\
\text { average }\end{array}$ & $\begin{array}{l}\text { Aver- } \\
\text { age }\end{array}$ & Average & High & $\begin{array}{l}\text { Aver- } \\
\text { age }\end{array}$ & $\begin{array}{c}\text { Below } \\
\text { average }\end{array}$ & $\begin{array}{c}\text { Below } \\
\text { average }\end{array}$ & $\begin{array}{c}\text { Below } \\
\text { average }\end{array}$ & $\begin{array}{c}\text { Below } \\
\text { average }\end{array}$ \\
\hline 6 & $\begin{array}{l}\text { Very } \\
\text { low }\end{array}$ & $\begin{array}{l}\text { Very } \\
\text { low }\end{array}$ & $\begin{array}{c}\text { Below } \\
\text { average }\end{array}$ & $\begin{array}{c}\text { Below } \\
\text { average }\end{array}$ & Average & $\begin{array}{c}\text { Above } \\
\text { average }\end{array}$ & $\begin{array}{l}\text { Very } \\
\text { high }\end{array}$ & $\begin{array}{c}\text { Below } \\
\text { average }\end{array}$ & $\begin{array}{c}\text { Below } \\
\text { average }\end{array}$ & $\begin{array}{c}\text { Above } \\
\text { average }\end{array}$ & $\begin{array}{c}\text { Above } \\
\text { average }\end{array}$ & $\begin{array}{l}\text { Aver- } \\
\text { age }\end{array}$ & $\begin{array}{c}\text { Below } \\
\text { average }\end{array}$ & Low & $\begin{array}{l}\text { Very } \\
\text { low }\end{array}$ \\
\hline 7 & Low & $\begin{array}{l}\text { Very } \\
\text { low }\end{array}$ & $\begin{array}{l}\text { Below } \\
\text { average }\end{array}$ & $\begin{array}{l}\text { Aver- } \\
\text { age }\end{array}$ & $\begin{array}{c}\text { Below } \\
\text { average }\end{array}$ & $\begin{array}{l}\text { Aver- } \\
\text { age }\end{array}$ & $\begin{array}{l}\text { Below } \\
\text { average }\end{array}$ & $\begin{array}{c}\text { Below } \\
\text { average }\end{array}$ & $\begin{array}{c}\text { Above } \\
\text { average }\end{array}$ & $\begin{array}{l}\text { Aver- } \\
\text { age }\end{array}$ & $\begin{array}{c}\text { Below } \\
\text { average }\end{array}$ & Low & Low & $\begin{array}{l}\text { Very } \\
\text { low }\end{array}$ & $\begin{array}{l}\text { Very } \\
\text { low }\end{array}$ \\
\hline 8 & $\begin{array}{c}\text { Below } \\
\text { average }\end{array}$ & Low & High & $\begin{array}{l}\text { Aver- } \\
\text { age }\end{array}$ & Average & $\begin{array}{l}\text { Below } \\
\text { average }\end{array}$ & Average & High & $\begin{array}{l}\text { Very } \\
\text { high }\end{array}$ & $\begin{array}{l}\text { Very } \\
\text { high }\end{array}$ & $\begin{array}{l}\text { Aver- } \\
\text { age }\end{array}$ & Low & $\begin{array}{l}\text { Very } \\
\text { low }\end{array}$ & $\begin{array}{c}\text { Below } \\
\text { average }\end{array}$ & $\begin{array}{l}\text { Very } \\
\text { low }\end{array}$ \\
\hline 9 & High & $\begin{array}{c}\text { Above } \\
\text { average }\end{array}$ & $\begin{array}{c}\text { Above } \\
\text { average }\end{array}$ & $\begin{array}{l}\text { Above } \\
\text { average }\end{array}$ & Average & High & $\begin{array}{c}\text { Above } \\
\text { average }\end{array}$ & High & $\begin{array}{c}\text { Ex- } \\
\text { tremely }\end{array}$ & $\begin{array}{l}\text { Below } \\
\text { average }\end{array}$ & $\begin{array}{c}\text { Below } \\
\text { average }\end{array}$ & $\begin{array}{l}\text { Very } \\
\text { low }\end{array}$ & Low & Average & $\begin{array}{c}\text { Ex- } \\
\text { tremely }\end{array}$ \\
\hline 10 & $\begin{array}{l}\text { Very } \\
\text { low }\end{array}$ & $\begin{array}{l}\text { Very } \\
\text { low }\end{array}$ & Low & $\begin{array}{l}\text { Very } \\
\text { low }\end{array}$ & $\begin{array}{c}\text { Ex- } \\
\text { tremely }\end{array}$ & $\begin{array}{l}\text { Aver- } \\
\text { age }\end{array}$ & $\begin{array}{l}\text { Above } \\
\text { average }\end{array}$ & Low & $\begin{array}{c}\text { Below } \\
\text { average }\end{array}$ & Low & $\begin{array}{c}\text { Above } \\
\text { average }\end{array}$ & $\begin{array}{l}\text { Very } \\
\text { low }\end{array}$ & $\begin{array}{l}\text { Very } \\
\text { low }\end{array}$ & $\begin{array}{c}\text { Ex- } \\
\text { tremely }\end{array}$ & $\begin{array}{c}\text { Ex- } \\
\text { tremely }\end{array}$ \\
\hline 11 & Average & $\begin{array}{c}\text { Above } \\
\text { average }\end{array}$ & Average & Low & $\begin{array}{l}\text { Very } \\
\text { low }\end{array}$ & High & $\begin{array}{c}\text { Ex- } \\
\text { tremely }\end{array}$ & $\begin{array}{l}\text { Very } \\
\text { low }\end{array}$ & $\begin{array}{c}\text { Above } \\
\text { average }\end{array}$ & $\begin{array}{l}\text { Very } \\
\text { low }\end{array}$ & $\begin{array}{l}\text { Aver- } \\
\text { age }\end{array}$ & $\begin{array}{l}\text { Very } \\
\text { low }\end{array}$ & $\begin{array}{c}\text { Ex- } \\
\text { tremely }\end{array}$ & Average & $\begin{array}{c}\text { Ex- } \\
\text { tremely }\end{array}$ \\
\hline 12 & Average & $\begin{array}{c}\text { Above } \\
\text { average }\end{array}$ & High & $\begin{array}{c}\text { Above } \\
\text { average }\end{array}$ & Low & $\begin{array}{l}\text { Very } \\
\text { high }\end{array}$ & $\begin{array}{c}\text { Above } \\
\text { average }\end{array}$ & $\begin{array}{c}\text { Below } \\
\text { average }\end{array}$ & Average & Low & $\begin{array}{l}\text { Aver- } \\
\text { age }\end{array}$ & $\begin{array}{l}\text { Very } \\
\text { low }\end{array}$ & $\begin{array}{c}\text { Below } \\
\text { average }\end{array}$ & High & Average \\
\hline 13 & $\begin{array}{c}\text { Above } \\
\text { average }\end{array}$ & Average & High & $\begin{array}{l}\text { Aver- } \\
\text { age }\end{array}$ & Average & $\begin{array}{c}\text { Above } \\
\text { average }\end{array}$ & Average & $\begin{array}{l}\text { Aver- } \\
\text { age }\end{array}$ & Average & $\begin{array}{c}\text { Above } \\
\text { average }\end{array}$ & $\begin{array}{l}\text { Aver- } \\
\text { age }\end{array}$ & $\begin{array}{c}\text { Above } \\
\text { average }\end{array}$ & $\begin{array}{c}\text { Below } \\
\text { average }\end{array}$ & Average & Average \\
\hline 14 & High & $\begin{array}{c}\text { Above } \\
\text { average }\end{array}$ & Average & $\begin{array}{l}\text { Aver- } \\
\text { age }\end{array}$ & Average & $\begin{array}{l}\text { Aver- } \\
\text { age }\end{array}$ & $\begin{array}{l}\text { Above } \\
\text { average }\end{array}$ & $\begin{array}{l}\text { Aver- } \\
\text { age }\end{array}$ & $\begin{array}{c}\text { Above } \\
\text { average }\end{array}$ & High & $\begin{array}{l}\text { Aver- } \\
\text { age }\end{array}$ & $\begin{array}{c}\text { Below } \\
\text { average }\end{array}$ & Low & Low & $\begin{array}{l}\text { Very } \\
\text { low }\end{array}$ \\
\hline 15 & Average & Average & $\begin{array}{c}\text { Above } \\
\text { average }\end{array}$ & $\begin{array}{l}\text { Aver- } \\
\text { age }\end{array}$ & $\begin{array}{c}\text { Below } \\
\text { average }\end{array}$ & $\begin{array}{l}\text { Aver- } \\
\text { age }\end{array}$ & $\begin{array}{l}\text { Above } \\
\text { average }\end{array}$ & $\begin{array}{l}\text { Below } \\
\text { average }\end{array}$ & Average & $\begin{array}{l}\text { Aver- } \\
\text { age }\end{array}$ & $\begin{array}{c}\text { Above } \\
\text { average }\end{array}$ & Low & $\begin{array}{l}\text { Very } \\
\text { low }\end{array}$ & $\begin{array}{l}\text { Very } \\
\text { low }\end{array}$ & $\begin{array}{c}\text { Ex- } \\
\text { tremely }\end{array}$ \\
\hline
\end{tabular}

Qualitative values of attributes 
Table 4

Crisp score of attributes

\begin{tabular}{|c|c|c|c|c|c|c|c|c|c|c|c|c|c|c|c|}
\hline & 1 & 2 & 3 & 4 & 5 & 6 & 7 & 8 & 9 & 10 & 11 & 12 & 13 & 14 & 15 \\
\hline 1 & 0.865 & 0.665 & 0.665 & 0.5 & 0.59 & 0.5 & 0.41 & 0.59 & 0.665 & 0.665 & 0.59 & 0.335 & 0.255 & 0.5 & 0.41 \\
\hline 2 & 0.41 & 0.41 & 0.665 & 0.5 & 0.255 & 0.5 & 0.59 & 0.59 & 0.41 & 0.41 & 0.665 & 0.59 & 0.5 & 0.41 & 0.5 \\
\hline 3 & 0.665 & 0.5 & 0.59 & 0.59 & 0.5 & 0.5 & 0.41 & 0.59 & 0.5 & 0.5 & 0.59 & 0.5 & 0.5 & 0.41 & 0.5 \\
\hline 4 & 0.745 & 0.865 & 0.665 & 0.59 & 0.41 & 0.5 & 0.41 & 0.59 & 0.59 & 0.5 & 0.665 & 0.41 & 0.41 & 0.5 & 0.41 \\
\hline 5 & 0.41 & 0.41 & 0.41 & 0.5 & 0.5 & 0.59 & 0.59 & 0.5 & 0.5 & 0.665 & 0.5 & 0.41 & 0.41 & 0.41 & 0.41 \\
\hline 6 & 0.255 & 0.255 & 0.41 & 0.41 & 0.5 & 0.59 & 0.745 & 0.41 & 0.41 & 0.59 & 0.59 & 0.5 & 0.41 & 0.335 & 0.255 \\
\hline 7 & 0.335 & 0.255 & 0.41 & 0.5 & 0.41 & 0.5 & 0.41 & 0.41 & 0.59 & 0.5 & 0.41 & 0.335 & 0.335 & 0.255 & 0.255 \\
\hline 8 & 0.41 & 0.335 & 0.665 & 0.5 & 0.5 & 0.41 & 0.5 & 0.665 & 0.745 & 0.745 & 0.5 & 0.335 & 0.255 & 0.41 & 0.255 \\
\hline 9 & 0.665 & 0.59 & 0.59 & 0.59 & 0.5 & 0.665 & 0.59 & 0.665 & 0.865 & 0.41 & 0.41 & 0.255 & 0.335 & 0.5 & 0.135 \\
\hline 10 & 0.255 & 0.255 & 0.335 & 0.255 & 0.135 & 0.5 & 0.59 & 0.335 & 0.41 & 0.335 & 0.59 & 0.255 & 0.255 & 0.135 & 0.135 \\
\hline 11 & 0.5 & 0.59 & 0.5 & 0.335 & 0.255 & 0.665 & 0.135 & 0.255 & 0.59 & 0.255 & 0.5 & 0.255 & 0.135 & 0.5 & 0.135 \\
\hline 12 & 0.5 & 0.59 & 0.665 & 0.59 & 0.335 & 0.745 & 0.59 & 0.41 & 0.5 & 0.335 & 0.5 & 0.255 & 0.41 & 0.665 & 0.5 \\
\hline 13 & 0.59 & 0.5 & 0.665 & 0.5 & 0.5 & 0.59 & 0.5 & 0.5 & 0.5 & 0.59 & 0.5 & 0.59 & 0.41 & 0.5 & 0.5 \\
\hline 14 & 0.665 & 0.59 & 0.5 & 0.5 & 0.5 & 0.5 & 0.59 & 0.5 & 0.59 & 0.665 & 0.5 & 0.41 & 0.335 & 0.335 & 0.255 \\
\hline 15 & 0.5 & 0.5 & 0.59 & 0.5 & 0.41 & 0.5 & 0.59 & 0.41 & 0.5 & 0.5 & 0.59 & 0.335 & 0.255 & 0.255 & 0.135 \\
\hline
\end{tabular}

Step 2: Standardization

All attributes are the beneficial attributes. The standardization of attribute data is carried out using equations 3 to 7 . The standardized decision matrix is shown in Table 5.

Table 5

Standardized decision matrix

\begin{tabular}{ccccccccccccccccccc}
\hline & 1 & 2 & 3 & 4 & 5 & 6 & 7 & 8 & 9 & 10 & 11 & 12 & 13 & 14 & 15 \\
\hline 1 & 2.00 & 1.07 & 0.97 & 0.10 & 1.41 & -0.59 & -0.73 & 0.81 & 0.87 & 1.11 & 0.66 & -0.44 & -0.94 & 0.73 & 0.63 \\
2 & -0.62 & -0.47 & 0.97 & 0.10 & -1.37 & -0.59 & 0.58 & 0.81 & -1.20 & -0.73 & 1.64 & 1.82 & 1.55 & 0.02 & 1.26 \\
3 & 0.85 & 0.08 & 0.31 & 1.08 & 0.67 & -0.59 & -0.73 & 0.81 & -0.47 & -0.08 & 0.66 & 1.02 & 1.55 & 0.02 & 1.26 \\
4 & 1.31 & 2.27 & 0.97 & 1.08 & -0.08 & -0.59 & -0.73 & 0.81 & 0.26 & -0.08 & 1.64 & 0.23 & 0.64 & 0.73 & 0.63 \\
5 & -0.62 & -0.47 & -1.28 & 0.10 & 0.67 & 0.47 & 0.58 & 0.05 & -0.47 & 1.11 & -0.52 & 0.23 & 0.64 & 0.02 & 0.63 \\
6 & -1.52 & -1.40 & -1.28 & -0.88 & 0.67 & 0.47 & 1.72 & -0.72 & -1.20 & 0.57 & 0.66 & 1.02 & 0.64 & -0.58 & -0.45 \\
7 & -1.06 & -1.40 & -1.28 & 0.10 & -0.08 & -0.59 & -0.73 & -0.72 & 0.26 & -0.08 & -1.71 & -0.44 & -0.13 & -1.21 & -0.45 \\
8 & -0.62 & -0.92 & 0.97 & 0.10 & 0.67 & -1.65 & -0.07 & 1.45 & 1.52 & 1.69 & -0.52 & -0.44 & -0.94 & 0.02 & -0.45 \\
9 & 0.85 & 0.62 & 0.31 & 1.08 & 0.67 & 1.35 & 0.58 & 1.45 & 2.50 & -0.73 & -1.71 & -1.15 & -0.13 & 0.73 & -1.29 \\
10 & -1.52 & -1.40 & -1.94 & -2.57 & -2.37 & -0.59 & 0.58 & -1.36 & -1.20 & -1.27 & 0.66 & -1.15 & -0.94 & -2.16 & -1.29 \\
11 & -0.10 & 0.62 & -0.48 & -1.70 & -1.37 & 1.35 & -2.74 & -2.04 & 0.26 & -1.85 & -0.52 & -1.15 & -2.15 & 0.73 & -1.29 \\
12 & -0.10 & 0.62 & 0.97 & 1.08 & -0.71 & 2.28 & 0.58 & -0.72 & -0.47 & -1.27 & -0.52 & -1.15 & 0.64 & 2.03 & 1.26 \\
13 & 0.42 & 0.08 & 0.97 & 0.10 & 0.67 & 0.47 & -0.07 & 0.05 & -0.47 & 0.57 & -0.52 & 1.82 & 0.64 & 0.73 & 1.26 \\
14 & 0.85 & 0.62 & -0.48 & 0.10 & 0.67 & -0.59 & 0.58 & 0.05 & 0.26 & 1.11 & -0.52 & 0.23 & -0.13 & -0.58 & -0.45 \\
15 & -0.10 & 0.08 & 0.31 & 0.10 & -0.08 & -0.59 & 0.58 & -0.72 & -0.47 & -0.08 & 0.66 & -0.44 & -0.94 & -1.21 & -1.29 \\
\hline & & & & & & & & & & & & & \\
\hline
\end{tabular}

Step 3: Ideal and anti-ideal points

Ideal points and anti-ideal points are the highest and lowest values of the attributes which is obtained from standardized decision matrix and shown in Table 6.

Table 6

Ideal point

\begin{tabular}{|c|c|c|c|c|c|c|c|c|c|c|c|c|c|c|c|}
\hline $\mathrm{a}^{*}$ & 2 & 2.27 & 0.97 & 1.08 & 1.41 & 2.28 & 1.72 & 1.45 & 2.5 & 1.69 & 1.64 & 1.82 & 1.55 & 2.03 & 1.26 \\
\hline $\mathrm{b}^{*}$ & -1.52 & -1.4 & -1.94 & -2.57 & -2.37 & -1.65 & -2.74 & -2.04 & -1.2 & -1.85 & -1.71 & -1.15 & -2.15 & -2.16 & -1.29 \\
\hline
\end{tabular}

Step 4: Attribute weights

The objective weights are calculated by using entropy method and the weights obtained are given in Table 7. The subjective weights of the attributes considered by Jain and Raj (2013c) are considered here for comparison purpose and these are given in Table 8.

Table 7

Objective weights

\begin{tabular}{llllllllllllllll}
\hline wj entropy, wo & 0.095 & 0.099 & 0.036 & 0.032 & 0.077 & 0.018 & 0.072 & 0.05 & 0.041 & 0.063 & 0.018 & 0.068 & 0.072 & 0.086 & 0.176
\end{tabular}


Table 8

Subjective weights

\begin{tabular}{llllllllllllllll}
\hline wj AHP, ws & 0.086 & 0.085 & 0.078 & 0.136 & 0.067 & 0.121 & 0.096 & 0.035 & 0.095 & 0.053 & 0.019 & 0.037 & 0.030 & 0.027 & 0.035
\end{tabular}

The integrated weights $\left(\mathrm{w}^{\mathrm{I}}\right)$ are calculated using equation 13 and the integrated weights are shown in

Table 9.

Table 9

Integrated weights

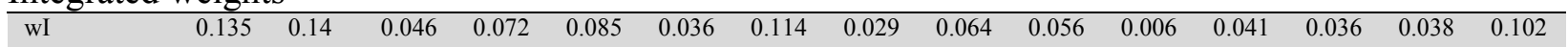

Step 5: WED, index score and ranking

The WEDs of an alternative from ideal points and anti-ideal points are calculated using equation 14 and 15 respectively and the index score is calculated using equation 16 . The index score values considering to all sets of weights are given in Table 10. The ranks of alternatives considering to different sets of weights i.e. subjective, objective and integrated are given in Table 11.

Table 10

Index score

\begin{tabular}{llllllllllllllll}
\hline WED + & 0.538 & 0.766 & 0.633 & 0.549 & 0.714 & 0.889 & 0.936 & 0.795 & 0.613 & 1.088 & 0.992 & 0.621 & 0.605 & 0.583 & 0.748 \\
WED- & 0.628 & 0.406 & 0.496 & 0.658 & 0.370 & 0.374 & 0.218 & 0.358 & 0.520 & 0.232 & 0.392 & 0.494 & 0.471 & 0.455 & 0.301 \\
Index Score & 0.539 & 0.346 & 0.439 & 0.545 & 0.341 & 0.296 & 0.189 & 0.31 & 0.459 & 0.176 & 0.283 & 0.443 & 0.438 & 0.438 & 0.287 \\
\hline
\end{tabular}

Table 11

Ranking of flexibility

\begin{tabular}{|c|c|c|c|c|c|c|c|}
\hline Sr. No. & Flexibility & WI & Rank & $\mathrm{WO}$ & Rank & WS & Rank \\
\hline 1 & Machine & 0.539 & 2 & 0.531 & 4 & 0.47 & 4 \\
\hline 2 & Routing & 0.346 & 8 & 0.483 & 6 & 0.347 & 10 \\
\hline 3 & Process & 0.439 & 5 & 0.539 & 3 & 0.43 & 6 \\
\hline 4 & Product & 0.545 & 1 & 0.542 & 2 & 0.497 & 3 \\
\hline 5 & Volume & 0.341 & 9 & 0.43 & 7 & 0.375 & 9 \\
\hline 6 & Material handling & 0.296 & 11 & 0.313 & 11 & 0.322 & 13 \\
\hline 7 & Operation & 0.189 & 14 & 0.213 & 13 & 0.265 & 14 \\
\hline 8 & Expansion & 0.31 & 10 & 0.327 & 10 & 0.379 & 8 \\
\hline 9 & Production & 0.459 & 3 & 0.348 & 9 & 0.594 & 1 \\
\hline 10 & Programme & 0.176 & 15 & 0.151 & 15 & 0.186 & 15 \\
\hline 11 & Market & 0.283 & 13 & 0.237 & 12 & 0.327 & 12 \\
\hline 12 & Respone & 0.443 & 4 & 0.521 & 5 & 0.549 & 2 \\
\hline 13 & Product mix & 0.438 & 6 & 0.552 & 1 & 0.441 & 5 \\
\hline 14 & Size & 0.438 & 6 & 0.371 & 8 & 0.402 & 7 \\
\hline 15 & Range & 0.287 & 12 & 0.206 & 14 & 0.333 & 11 \\
\hline
\end{tabular}

In this method, from Table 11, according to integrated weight found that product flexibility (4) has got the rank one and programme flexibility (10) got fifteen rank by using integrated weights.

\section{Ranking of flexibility of FMS by MOORA Method:}

\section{(a) By Ratio system approach:}

To rank the flexibility of FMS the following steps are taken as given below:

Step 1: Decision matrix for flexibility of FMS is presented in Table 4.

Step 2: Decision matrix is normalized by using equation (17) as presented in Table 12.

Step 3: The attributes are classified into beneficial and non-beneficial attribute. Here, all the attribute are the beneficial attributes.

Step 4: The assessment value (yi) for each considered alternative is computed by using Eq. (18) as presented in Table 12. 
Table 12

Normalized decision matrix

\begin{tabular}{|c|c|c|c|c|c|c|c|c|c|c|c|c|c|c|c|c|}
\hline \multirow{2}{*}{ Sr. No } & \multirow{2}{*}{$\begin{array}{c}\text { Alternatives } \\
\text { (Flexibilities) }\end{array}$} & \multicolumn{15}{|c|}{ Attributes } \\
\hline & & 1 & 2 & 3 & 4 & 5 & 6 & 7 & 8 & 9 & 10 & 11 & 12 & 13 & 14 & 15 \\
\hline 1 & Machine & 0.111 & 0.091 & 0.080 & 0.068 & 0.094 & 0.061 & 0.054 & 0.080 & 0.079 & 0.087 & 0.073 & 0.058 & 0.049 & 0.082 & 0.086 \\
\hline 2 & Routing & 0.053 & 0.056 & 0.080 & 0.068 & 0.040 & 0.061 & 0.077 & 0.080 & 0.049 & 0.053 & 0.082 & 0.102 & 0.096 & 0.067 & 0.104 \\
\hline 3 & Process & 0.086 & 0.068 & 0.071 & 0.080 & 0.079 & 0.061 & 0.054 & 0.080 & 0.060 & 0.065 & 0.073 & 0.087 & 0.096 & 0.067 & 0.104 \\
\hline 4 & Product & 0.096 & 0.118 & 0.080 & 0.080 & 0.065 & 0.061 & 0.054 & 0.080 & 0.071 & 0.065 & 0.082 & 0.071 & 0.079 & 0.082 & 0.086 \\
\hline 5 & Volume & 0.053 & 0.056 & 0.049 & 0.068 & 0.079 & 0.071 & 0.077 & 0.067 & 0.060 & 0.087 & 0.062 & 0.071 & 0.079 & 0.067 & 0.086 \\
\hline 6 & Material handling & 0.033 & 0.035 & 0.049 & 0.056 & 0.079 & 0.071 & 0.097 & 0.055 & 0.049 & 0.077 & 0.073 & 0.087 & 0.079 & 0.055 & 0.053 \\
\hline 7 & Operation & 0.043 & 0.035 & 0.049 & 0.068 & 0.065 & 0.061 & 0.054 & 0.055 & 0.071 & 0.065 & 0.051 & 0.058 & 0.064 & 0.042 & 0.053 \\
\hline 8 & Expansion & 0.053 & 0.046 & 0.080 & 0.068 & 0.079 & 0.050 & 0.065 & 0.090 & 0.089 & 0.097 & 0.062 & 0.058 & 0.049 & 0.067 & 0.053 \\
\hline 9 & Production & 0.086 & 0.081 & 0.071 & 0.080 & 0.079 & 0.081 & 0.077 & 0.090 & 0.103 & 0.053 & 0.051 & 0.044 & 0.064 & 0.082 & 0.028 \\
\hline 10 & Programme & 0.033 & 0.035 & 0.040 & 0.035 & 0.021 & 0.061 & 0.077 & 0.045 & 0.049 & 0.044 & 0.073 & 0.044 & 0.049 & 0.022 & 0.028 \\
\hline 11 & Market & 0.064 & 0.081 & 0.060 & 0.046 & 0.040 & 0.081 & 0.018 & 0.034 & 0.071 & 0.033 & 0.062 & 0.044 & 0.026 & 0.082 & 0.028 \\
\hline 12 & Response & 0.064 & 0.081 & 0.080 & 0.080 & 0.053 & 0.090 & 0.077 & 0.055 & 0.060 & 0.044 & 0.062 & 0.044 & 0.079 & 0.109 & 0.104 \\
\hline 13 & Product mix & 0.076 & 0.068 & 0.080 & 0.068 & 0.079 & 0.071 & 0.065 & 0.067 & 0.060 & 0.077 & 0.062 & 0.102 & 0.079 & 0.082 & 0.104 \\
\hline 14 & Size & 0.086 & 0.081 & 0.060 & 0.068 & 0.079 & 0.061 & 0.077 & 0.067 & 0.071 & 0.087 & 0.062 & 0.071 & 0.064 & 0.055 & 0.053 \\
\hline 15 & Range flexibility & 0.064 & 0.068 & 0.071 & 0.068 & 0.065 & 0.061 & 0.077 & 0.055 & 0.060 & 0.065 & 0.073 & 0.058 & 0.049 & 0.042 & 0.028 \\
\hline
\end{tabular}

Step 5: The relative importance of attributes is analyze with respect to the objective and it is taken as in AHP methodology. Weight of attributes are taken from (Jain \& Raj, 2013c) and shown in Table 13.

Table 13

Ranking of flexibility (without considering weights of attributes)

\begin{tabular}{cccc}
\hline Sr. No & $\begin{array}{c}\text { Alternatives } \\
\text { (Flexibilities) }\end{array}$ & $y_{i}$ & Ranking \\
\hline 1 & Machine & 1.151 & 2.000 \\
2 & Routing & 1.069 & 7.000 \\
3 & Process & 1.130 & 4.000 \\
4 & Product & 1.168 & 1.000 \\
5 & Volume & 1.032 & 9.000 \\
6 & Material handling & 0.948 & 11.000 \\
7 & Operation & 0.833 & 13.000 \\
8 & Expansion & 1.006 & 10.000 \\
9 & Production & 1.070 & 6.000 \\
10 & Programme & 0.656 & 15.000 \\
11 & Market & 0.769 & 14.000 \\
12 & Response & 1.082 & 5.000 \\
13 & Product mix & 1.141 & 3.000 \\
14 & Size & 1.041 & 8.000 \\
15 & Range flexibility & 0.904 & 12.000 \\
\hline
\end{tabular}

Step 6: The assessment value (yi) for each considered alternative with weight of attributes (Table 8) are computed by using equation (19) as presented in Table 14.

Table 14

Ranking of flexibility (considering weights of attributes)

Sr. No $\quad \begin{gathered}\text { Alternatives } \\ \text { (Flexibilities) }\end{gathered}$

$\mathrm{y}_{\mathrm{i} *} \quad$ Ranking

\begin{tabular}{llll}
\hline 1 & Machine & 0.081 & 2.000 \\
2 & Routing & 0.071 & 9.000 \\
\hline
\end{tabular}




\begin{tabular}{|cccc}
\hline 3 & Process & 0.076 & 6.000 \\
\hline 4 & Product & 0.081 & 1.000 \\
5 & Volume & 0.071 & 8.000 \\
6 & Material handling & 0.065 & 12.000 \\
7 & Operation & 0.059 & 13.000 \\
\hline 8 & Expansion & 0.070 & 10.000 \\
9 & Production & 0.079 & 3.000 \\
10 & Programme & 0.048 & 15.000 \\
11 & Market & 0.057 & 14.000 \\
\hline 12 & Response & 0.076 & 5.000 \\
13 & Product mix & 0.077 & 4.000 \\
\hline 14 & Size & 0.074 & 7.000 \\
\hline 15 & Range flexibility & 0.067 & 11.000 \\
\hline
\end{tabular}

From Table 13 and 14, product flexibility is the top flexibility.

\section{(b) By Reference point approach:}

Step 1: Decision matrix for flexibility of FMS is presented in Table 4 as in the ratio system approach.

Step 2: Decision matrix (Table 4) is normalized by using equation (17) as presented in Table 12 as in the ratio system approach.

Step 3: The attributes are classified into beneficial and non-beneficial attribute. Here, all the attribute are the beneficial attributes.

Step 4: Set the reference point as maximum value because all attributes are beneficial. The reference point of attributes are shown in Table 15. The deviation from reference point are calculated by using equation 22 and shown in table 16.

Table 15

Reference point of attributes

\begin{tabular}{|c|c|c|c|c|c|c|c|c|c|c|c|c|c|c|c|}
\hline Attributes & 1 & 2 & 3 & 4 & 5 & 6 & 7 & 8 & 9 & 10 & 11 & 12 & 13 & 14 & 15 \\
\hline $\begin{array}{c}\text { Reference } \\
\text { point }\end{array}$ & 0.111 & 0.118 & .080 & 0.080 & 0.094 & 0.090 & 0.097 & 0.090 & 0.103 & 0.097 & 0.082 & 0.102 & 0.096 & 0.109 & 0.104 \\
\hline
\end{tabular}

Table 16

Deviation from reference point of the attributes

\begin{tabular}{|c|c|c|c|c|c|c|c|c|c|c|c|c|c|c|c|c|}
\hline \multirow{2}{*}{ Sr. No } & \multirow{2}{*}{$\begin{array}{l}\text { Alternatives } \\
\text { (Flexibilities) }\end{array}$} & \multicolumn{15}{|c|}{ Attributes } \\
\hline & & 1 & 2 & 3 & 4 & 5 & 6 & 7 & 8 & 9 & 10 & 11 & 12 & 13 & 14 & 15 \\
\hline 1 & Machine & 0.000 & 0.027 & 0.000 & 0.012 & 0.000 & 0.030 & 0.044 & 0.010 & 0.024 & 0.010 & 0.009 & 0.044 & 0.047 & 0.027 & 0.019 \\
\hline 2 & Routing & 0.059 & 0.062 & 0.000 & 0.012 & 0.053 & 0.030 & 0.020 & 0.010 & 0.054 & 0.044 & 0.000 & 0.000 & 0.000 & 0.042 & 0.000 \\
\hline 3 & Process & 0.026 & 0.050 & 0.009 & 0.000 & 0.014 & 0.030 & 0.044 & 0.010 & 0.044 & 0.032 & 0.009 & 0.016 & 0.000 & 0.042 & 0.000 \\
\hline 4 & Product & 0.015 & 0.000 & 0.000 & 0.000 & 0.029 & 0.030 & 0.044 & 0.010 & 0.033 & 0.032 & 0.000 & 0.031 & 0.017 & 0.027 & 0.019 \\
\hline 5 & Volume & 0.059 & 0.062 & 0.031 & 0.012 & 0.014 & 0.019 & 0.020 & 0.022 & 0.044 & 0.010 & 0.020 & 0.031 & 0.017 & 0.042 & 0.019 \\
\hline 6 & Material handling & 0.079 & 0.083 & 0.031 & 0.024 & 0.014 & 0.019 & 0.000 & 0.034 & 0.054 & 0.020 & 0.009 & 0.016 & 0.017 & 0.054 & 0.051 \\
\hline 7 & Operation & 0.068 & 0.083 & 0.031 & 0.012 & 0.029 & 0.030 & 0.044 & 0.034 & 0.033 & 0.032 & 0.031 & 0.044 & 0.032 & 0.067 & 0.051 \\
\hline 8 & Expansion & 0.059 & 0.073 & 0.000 & 0.012 & 0.014 & 0.041 & 0.032 & 0.000 & 0.014 & 0.000 & 0.020 & 0.044 & 0.047 & 0.042 & 0.051 \\
\hline 9 & Production & 0.026 & 0.038 & 0.009 & 0.000 & 0.014 & 0.010 & 0.020 & 0.000 & 0.000 & 0.044 & 0.031 & 0.058 & 0.032 & 0.027 & 0.076 \\
\hline 10 & Programme & 0.079 & 0.083 & 0.040 & 0.046 & 0.072 & 0.030 & 0.020 & 0.044 & 0.054 & 0.053 & 0.009 & 0.058 & 0.047 & 0.087 & 0.076 \\
\hline 11 & Market & 0.047 & 0.038 & 0.020 & 0.035 & 0.053 & 0.010 & 0.080 & 0.055 & 0.033 & 0.064 & 0.020 & 0.058 & 0.070 & 0.027 & 0.076 \\
\hline 12 & Response & 0.047 & 0.038 & 0.000 & 0.000 & 0.040 & 0.000 & 0.020 & 0.034 & 0.044 & 0.053 & 0.020 & 0.058 & 0.017 & 0.000 & 0.000 \\
\hline 13 & Product mix & 0.035 & 0.050 & 0.000 & 0.012 & 0.014 & 0.019 & 0.032 & 0.022 & 0.044 & 0.020 & 0.020 & 0.000 & 0.017 & 0.027 & 0.000 \\
\hline 14 & Size & 0.026 & 0.038 & 0.020 & 0.012 & 0.014 & 0.030 & 0.020 & 0.022 & 0.033 & 0.010 & 0.020 & 0.031 & 0.032 & 0.054 & 0.051 \\
\hline 15 & Range flexibility & 0.047 & 0.050 & 0.009 & 0.012 & 0.029 & 0.030 & 0.020 & 0.034 & 0.044 & 0.032 & 0.009 & 0.044 & 0.047 & 0.067 & 0.076 \\
\hline
\end{tabular}


Step 5: Computation of performance index is done by using equation 23 and shown in Table 17.

Table 17

Ranking of flexibility by using performance index

\begin{tabular}{|c|c|c|c|}
\hline Sr. No & $\begin{array}{c}\text { Alternatives } \\
\text { (Flexibilities) }\end{array}$ & $\mathrm{P}_{\mathrm{i}}$ & Ranking \\
\hline 1 & Machine & 0.0470 & 2 \\
\hline 2 & Routing & 0.0622 & 7 \\
\hline 3 & Process & 0.0499 & 3 \\
\hline 4 & Product & 0.0438 & 1 \\
\hline 5 & Volume & 0.0622 & 7 \\
\hline 6 & Material handling & 0.0834 & 14 \\
\hline 7 & Operation & 0.0834 & 13 \\
\hline 8 & Expansion & 0.0725 & 9 \\
\hline 9 & Production & 0.0762 & 10 \\
\hline 10 & Programme & 0.0866 & 15 \\
\hline 11 & Market & 0.0797 & 12 \\
\hline 12 & Response & 0.0581 & 6 \\
\hline 13 & Product mix & 0.0499 & 3 \\
\hline 14 & Size & 0.0539 & 5 \\
\hline 15 & Range flexibility & 0.0762 & 10 \\
\hline
\end{tabular}

Step 6: Best alternative and ranking is shown in Table 17.

From Table 17, product flexibility has got rank one.

\section{Discussion and Conclusion}

The objective of this research is to focus on the ranking of fifteen flexibility which is identified in a flexible manufacturing system so that management may effectively deal with these flexibilities. In this research, ranking of flexibilities is taken by a WEDBA and MOORA approaches i.e. MADM methods. These approaches represents the qualitative attribute on a conversion scale using fuzzy logic to a quantitative attributes values. Objective weight of attributes have taken by entropy method and integrated weights have taken from both i.e. subjective and objective weights. Subjective weight of attributes have taken same as AHP method. By WEDBA got the ranking is 4-1-9-12-3-13-14-2-5-8-6-15-11-7-10. In this, product flexibility has the top ranking and programme flexibility has lower most ranking.

While MOORA as a MADM method is also applied for ranking of flexibility of FMS by two ways i.e. simple ratio analysis and reference point. Further ratio analysis is applied in two ways i.e. considering attributes and without considering attribute weights. Weights of attributes are taken as per AHP methodology. Ranking without considering the attributes weights product flexibility is the top most flexibility and programme flexibility is the last one. While considering the weights of attributes results are same as product flexibility is the top most flexibility and programme flexibility is the last one. After this reference point analysis is taken and the results are same as top one is product flexibility and programme flexibility is the last.

These methods are easy to use and simple for calculation purposes. So, computational time will be less. Special thing is that this method is not required any special parameters as required in VIKOR. So, finally concluded that these methods can be utilized for decision making environments effectively.

Ranking of flexibilities is shown in Fig. 5. 


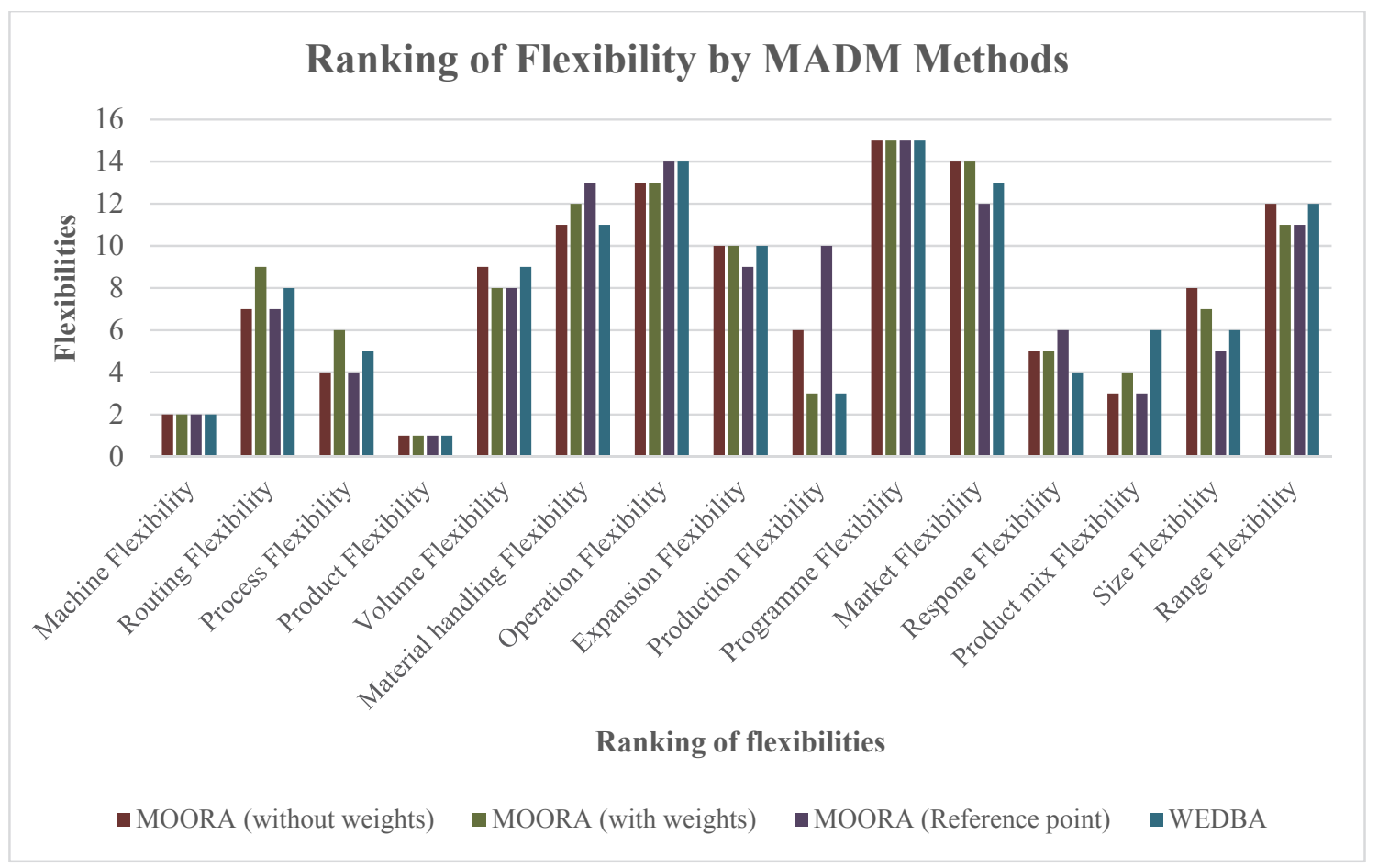

Fig. 5. Ranking of flexibility of FMS by MOORA and WEDBA method

It is concluded that product flexibility is the main flexibility in FMS. So, manager should focus on the product flexibility to increase the increase the flexibility of FMS and finally performance of FMS will increased as the flexibility is one of the factors of performance of FMS. Furthermore, there is a very high Spearman's rank correlation between the methods used. The Spearman's rank correlation coefficient between the methods are shown in Fig. 6. 


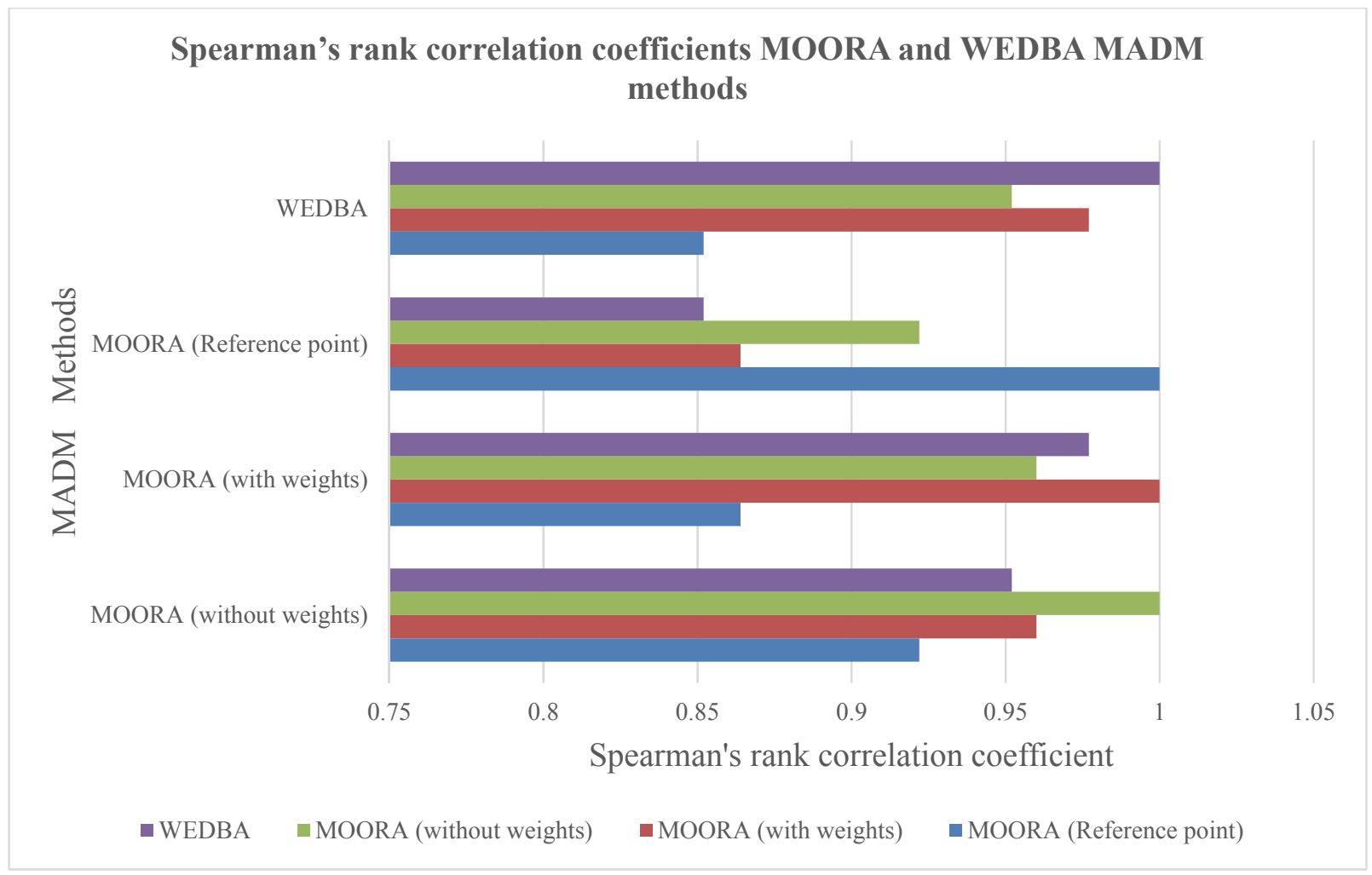

Fig. 6. Spearman's rank correlation coefficients between MOORA and WEDBA MADM methods for ranking of flexibility of FMS

There is no consistency in the result. The approaches are logical, simple and convenient to implement. These approaches can be extended to any other decision making situations of the manufacturing environment.

\section{References}

Attri, R., \& Grover, S. (2014). Decision making over the production system life cycle: MOORA method. International Journal of System Assurance Engineering and Management, 5(3), 320-328.

Brauers, W. K. (2004). Optimization Methods for a Stakeholder Society. Boston: Kluwer Academic.

Brauers, W. K. M. (2013). Multi-objective seaport planning by MOORA decision making. Annals of Operations Research, 206(1), 39-58.

Brauers, W. K. M., \& Zavadskas, E. K. (2006). The MOORA method and its application to privatization in a transition economy. Control and Cybernetics, 35(2), 445.

Brauers, W. K. M., \& Zavadskas, E. K. (2009). Robustness of the multi-objective MOORA method with a test for the facilities sector. Technological and Economic Development of Economy, 15(2), 352-375.

Brauers, W. K. M., \& Zavadskas, E. K. (2010). Project management by MULTIMOORA as an instrument for transition economies. Technological and Economic Development of Economy, 16(1), 5-24.

Brauers, W. K. M., \& Zavadskas, E. K. (2011). MULTIMOORA optimization used to decide on a bank loan to buy property. Technological and Economic Development of Economy, 17(1), 174-188.

Chakraborty, S. (2011). Applications of the MOORA method for decision making in manufacturing environment. The International Journal of Advanced Manufacturing Technology, 54(9-12), 1155-1166.

Chand, M., Raj, T., \& Shankar, R. (2014). A comparative study of multi criteria decision making approaches for risks assessment in supply chain. International Journal of Business Information Systems, 18(1), 67-84.

Chen, I. J., \& Chung, C. H. (1996). An examination of flexibility measurements and performance of flexible manufacturing systems. International Journal of Production Research, 34(2), 379-394.

Chen, S. J., \& Hwang, C. L. (1992). Fuzzy multiple attribute decision making methods. Heidelberg: Springer Berlin. 
Das, S. K. (1996). The measurement of flexibility in manufacturing systems. International Journal of Flexible Manufacturing Systems, 8(1), 67-93.

Dattorro, J. (2005). Convex optimization \& Euclidean distance geometry. USA: Meboo Publishing.

Gadakh, V., Shinde, V., \& Khemnar, N. (2013). Optimization of welding process parameters using MOORA method. The International Journal of Advanced Manufacturing Technology, 69(9-12), 2031-2039.

Ginevičius, R., Brauers, W. K. M., \& Podvezko, V. (2010). Regional development in Lithuania considering multiple objectives by the MOORA method. Technological and Economic Development of Economy(4), 613640.

Gower, J. C. (1982). Euclidean distance geometry. Math. Scientist, 7(1), 1-14.

Jain, V. (2018). Application of combined MADM methods as MOORA and PSI for ranking of FMS performance factors. Benchmarking: An International Journal, 25(6), 1903-1920.

Jain, V., \& Raj, T. (2013a). Evaluating the Variables Affecting Flexibility in FMS by Exploratory and Confirmatory Factor Analysis. Global Journal of Flexible Systems Management, 14(4), 181-193. doi: 10.1007/s40171-013-0042-9

Jain, V., \& Raj, T. (2013b). Evaluation of flexibility in FMS using SAW and WPM. Decision Science Letters, 2(4), 223-230.

Jain, V., \& Raj, T. (2013c). Ranking of Flexibility in Flexible Manufacturing System by Using a Combined Multiple Attribute Decision Making Method. Global Journal of Flexible Systems Management, 14(3), 125141. doi: http://dx.doi.org/10.1007/s40171-013-0038-5

Jain, V., \& Raj, T. (2014a). Evaluation of flexibility in FMS by VIKOR methodology. International Journal of Industrial and Systems Engineering, 18(4), 483-498.

Jain, V., \& Raj, T. (2014b). Modelling and analysis of FMS productivity variables by ISM, SEM and GTMA approach. Frontiers of Mechanical Engineering, 9(3), 218-232.

Jain, V., \& Raj, T. (2015a). Evaluating the intensity of variables affecting flexibility in FMS by graph theory and matrix approach. International Journal of Industrial and Systems Engineering, 19(2), 137-154.

Jain, V., \& Raj, T. (2015b). A hybrid approach using ISM and modified TOPSIS for the evaluation of flexibility in FMS. International Journal of Industrial and Systems Engineering, 19(3), 389-406.

Jain, V., \& Raj, T. (2015c). Modeling and analysis of FMS flexibility factors by TISM and fuzzy MICMAC. International Journal of System Assurance Engineering and Management, 6(3), 350-371.

Jain, V., \& Raj, T. (2016). Modeling and analysis of FMS performance variables by ISM, SEM and GTMA approach. International journal of production economics, 171(1), 84-96.

Jain, V., \& Raj, T. (2017). Tool life management of unmanned production system based on surface roughness by ANFIS. International Journal of System Assurance Engineering and Management, 8(2), 458-467.

Jain, V., \& Raj, T. (2018). Identification of performance variables which affect the FMS: a state-of-the-art review. International Journal of Process Management and Benchmarking, 8(4), 470-489.

Karande, P., \& Chakraborty, S. (2012). Application of multi-objective optimization on the basis of ratio analysis (MOORA) method for materials selection. Materials \& Design, 37, 317-324.

Raj, T., Attri, R., \& Jain, V. (2012). Modelling the factors affecting flexibility in FMS. International Journal of Industrial and Systems Engineering, 11(4), 350-374.

Rao, R. V. (2007). Decision making in the manufacturing environment: using graph theory and fuzzy multiple attribute decision making methods. London: Springer.

Rao, R. V. (2013). Decision Making in Manufacturing Environment Using Graph Theory and Fuzzy Multiple Attribute Decision Making Methods (Vol. 2). London: Springer-Verlag.

Rao, R. V., \& Singh, D. (2011). Evaluating flexible manufacturing systems using Euclidean distance-based integrated approach. International Journal of Decision Sciences, Risk and Management, 3(1-2), 32-53.

Rao, R. V., \& Singh, D. (2012). Weighted Euclidean distance based approach as a multiple attribute decision making method for plant or facility layout design selection. International Journal of Industrial Engineering Computations, 3(3), 365-382.

Saaty, T. L. (2000). Fundamentals of decision making and priority theory with the analytic hierarchy process (Vol. 6). Pittsburgh, PA: RWS Publications.

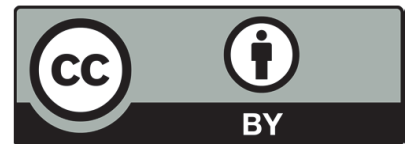

(C) 2018 by the authors; licensee Growing Science, Canada. This is an open access article distributed under the terms and conditions of the Creative Commons Attribution (CCBY) license (http://creativecommons.org/licenses/by/4.0/). 